\title{
DESIGN OF THE BEST SPACE-TIME TRELLIS CODES BASED ON COSET PARTITIONING
}

\author{
Pierre Viland, Gheorghe Zaharia and Jean-Francois Hélard \\ Institute for Electronics and Telecommunications of Rennes (IETR) - UMR CNRS 6164 \\ INSA -20 avenue des Buttes de Coësmes, \\ 35043 Rennes, France \\ Email: pierre.viland@insa-rennes.fr
}

\begin{abstract}
Several criteria to design good space-time trellis codes (STTCs) have been developed but an exhaustive search is still required to obtain the best codes. In this paper, we propose a new and fast method to design the best STTCs for $2^{n}$-PSK modulations. This method is an extension of the set partitioning proposed by Ungerboeck applied for multiple input multiple output (MIMO) systems. Based on this design method, new 4-PSK and 8-PSK codes are proposed and compared to the best known codes.
\end{abstract}

\section{INTRODUCTION}

The MIMO systems use antenna arrays at both sides of a radio link to drastically improve the reliability and/or the data rate of the wireless communication. In order to exploit the performance of the MIMO systems, several channel coding techniques have been proposed. For example, the STTCs combine the spatial diversity and time diversity in order to combat efficiently the signal fading. The STTCs have been proposed by Tarokh et al. in [1], where the analytic bound and the first design criteria have been obtained. The rank and the determinant criteria have been proposed to design good codes in the case of slow Rayleigh fading channels. For fast Rayleigh fading channels, the product distance and the Hamming distance criteria have been proposed. In [2], Vucetic et al. proposed the trace criterion for a slow Rayleigh fading channel. Based on the Euclidean distance, this criterion governs the performance for a great product of the number of transmit and receive antennas.

Thanks to these criteria, many 4-PSK and 8-PSK codes have been proposed to decrease the bit and frame error rate [1-5]. However, a systematic search has been required to find these codes. The aim of this paper is to propose a new method to design the best $2^{n}$-PSK codes with $n_{T}$ transmit antennas. This method is an extension of the set partitioning proposed by Ungerboeck [6-8]. In fact, he states the following rules to design a trellis-coded modulation (TCM):

1. Each point of the constellation has the same number of occurrences.
2. In the trellis, transitions originating from the same state or merging into the same state should be assigned subsets which contain signal points separated by the largest Euclidean distance.

3. Parallel paths should be assigned signal points separated by the largest Euclidean distance. Since there are no parallel paths in the STTCs, this rule is not important for the new design of the STTCs.

Thanks to this new method called 'coset partitioning', it is possible to design the best STTCs without an exhaustive search. The paper is organised as follows. Section II describes the representations of space-time codes and the existing design criteria. In section III, the new method is presented. In the last section, new 4-PSK and 8-PSK are given and their performance is compared to the best known codes.

\section{SYSTEM MODEL}

\subsection{Representation of the space-time trellis encoder}

We consider a $2^{n}$-PSK space-time trellis encoder with $n_{T}$ transmit antennas and $n_{R}$ receive antennas. For $n=2$, the encoder is shown in Fig. 1.

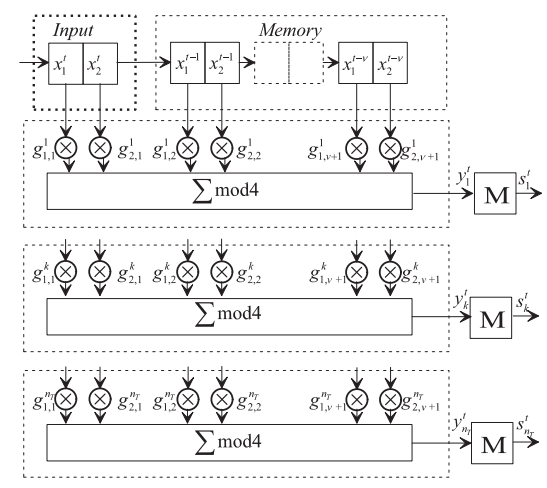

Fig. 1. Space-time trellis encoder for $4-\mathrm{PSK}$ and $n_{T}$ transmit antennas 
The encoder is composed of one input block of $n$ bits and $\nu$ memory blocks of $n$ bits. The state is defined by the binary values of the $\nu$ memory blocks of $n$ bits. At each time $t \in \mathbb{Z}$, all the bits of a block are replaced by the $n$ bits of the previous block. The $i^{t h}$ bit of the $j^{t h}$ block $x_{i}^{t-j+1}$, with $i=1 \ldots n$ and $j=1 \ldots \nu+1$, is associated to $n_{T}$ multiplier coefficients $g_{i, j}^{k} \in \mathbb{Z}_{2^{n}}, k=1 \ldots n_{T}$, where $n_{T}$ is the number of transmit antennas. With these $n_{T} \times n(\nu+1)$ coefficients, we define a generator matrix $G$ with $n_{T}$ lines and $\nu+1$ blocks of $n$ columns:

$$
\boldsymbol{G}=\left[G_{1}^{1} \ldots G_{n}^{1}|\ldots| G_{1}^{\nu+1} \ldots G_{n}^{\nu+1}\right]
$$

with $G_{i}^{j}=\left[g_{i, j}^{1} \ldots g_{i, j}^{n_{T}}\right]^{T} \in \mathbb{Z}_{2^{n}}^{n_{T}}$. At each time $t$, the encoder output $\boldsymbol{y}_{x^{t}}=\left[y_{1}^{t} y_{2}^{t} \ldots y_{n_{T}}^{t}\right]^{T} \in \mathbb{Z}_{2^{n}}^{n_{T}}$ is given by

$$
\boldsymbol{y}_{x^{t}}=\boldsymbol{G} \boldsymbol{x}^{t}
$$

where $x^{t}=\left[x_{1,1}^{t} \ldots x_{1, n}^{t} \ldots x_{\nu+1,1}^{t} \ldots x_{\nu+1, n}^{t}\right]^{T}$ is the extendedstate at time $t$ of the $L=n(\nu+1)$ length shift register realized by the input block followed by the $\nu$ memory blocks of $n$ bits.

Each encoder output $y_{k}^{t}$ is mapped onto a $2^{n}$-PSK signal given by: $s_{k}^{t}=\exp \left(j \frac{\pi}{2^{n-1}} y_{k}^{t}\right)$. Each output signal $s_{k}^{t}$ is sent to the $k^{t h}$ transmit antenna. At each time $t$, the set of symbols transmited simultaneously over the fading MIMO channel is given by $\boldsymbol{s}^{t}=\left[s_{1}^{t} s_{2}^{t} \ldots s_{n_{T}}^{t}\right]^{T}$.

An encoder can also be represented by a trellis, as shown in Fig. 2 for 4-PSK 4 states STTC.

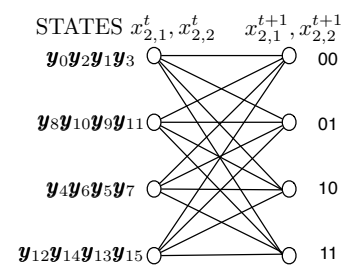

Fig. 2. 4-PSK 4 states STTC

In the trellis, the states are described by the points and the transitions between the states by the lines. Each transition corresponds to an extended-state. The vector $\boldsymbol{y}_{i} \in \mathbb{Z}_{4}^{n_{T}}$ represents the MIMO symbol associated to an extended-state. The index $i$ is computed as the decimal value of this extendedstate, where $x_{1,1}^{t}$ is the least significant bit. In this example, the trellis representation corresponds to the generator matrix $\boldsymbol{G}=\left[y_{1} y_{2} \mid y_{4} y_{8}\right]$.

In the general case, for a $2^{n}$-PSK STTC, there are $2^{n}$ transitions originating from the same state or merging into the same state. Each MIMO symbol belongs to $\mathbb{Z}_{2^{n}}^{n_{T}}$.

\subsection{Design criteria}

The main design criteria have been established in $[1,2]$ in order to decrease the bit and frame error rate. In this paper, only the case of slow fading channels is considered. Hence, the fading coefficients are constant during one frame of $L_{f}$ symbols. Besides, we assume that the decoder uses a maximum likelihood to estimate the transmitted symbols.

The main goal of this method is to reduce the pairwise error probability (PEP) which is the probability that the decoder selects an erroneous sequence. It is possible to represent the $L_{f}$ length transmitted frame beginning at time $t=0$ by a $n_{T} \times L_{f}$ dimension matrix $S=\left[\boldsymbol{s}^{0} \boldsymbol{s}^{1} \ldots \boldsymbol{s}^{L_{f}-1}\right]$. An error occurs if the decoder decides that an another frame $\boldsymbol{E}=\left[\boldsymbol{e}^{0} \boldsymbol{e}^{1} \ldots \boldsymbol{e}^{L_{f}-1}\right]$ is transmitted. Let us define the $n_{T} \times L_{f}$ difference matrix $\boldsymbol{B}=\boldsymbol{E}-\boldsymbol{S}$. The $n_{T} \times n_{T}$ product matrix $\boldsymbol{A}=\boldsymbol{B} \boldsymbol{B}^{*}$ is introduced, where $\boldsymbol{B}^{*}$ denotes the hermitian of $\boldsymbol{B}$. We define $r=\min (\operatorname{rank}(\boldsymbol{B}))$ where $\boldsymbol{B}$ is computed for all pairs of different coded frames $(\boldsymbol{E}, \boldsymbol{S})$. The design criteria depend on the value of the product $r n_{R}$.

The product $r n_{R} \leq 3$ : two criteria have been proposed $[1,9]$ to reduce the PEP. $\boldsymbol{A}$ has to be a full rank for any pair $(\boldsymbol{E}, \boldsymbol{S})$ and the coding gain given by $\eta=\sum_{d} N(d) d^{-n_{R}}$ must be minimized. $N(d)$ is defined as the average number of error events with determinant $d=\operatorname{det}(A)$.

The product $r n_{R} \geq 4$ : it has been shown in [2] that the PEP is minimized if the minimum trace of $A$ computed for all pairs $(\boldsymbol{E}, \boldsymbol{S})$ with $\boldsymbol{E} \neq \boldsymbol{S}$ is maximized.

In this paper we consider the case $r n_{R} \geq 4$ which is obtained if the rank of the generator matrix $G$ is greater than 1 and if there are at least 2 receive antennas.

\section{COSET PARTITIONING FOR SPACE-TIME TRELLIS CODES}

\subsection{Coset decomposition}

The MIMO symbols belong to the group $\mathbb{Z}_{2^{n}}^{n_{T}}$. This group can be decomposed in cosets as in [10]:

$$
\mathbb{Z}_{2^{n}}^{n_{T}}=\bigcup_{g \in\left[\mathbb{Z}_{2^{n}}^{n_{T}} / C_{0}\right]}\left(g+C_{0}\right)
$$

where $C_{0}=2^{n-1} \mathbb{Z}_{2}^{n_{T}}$ is a normal subgroup of $\mathbb{Z}_{2^{n}}^{n_{T}}$ such as $\forall v \in C_{0}, v=-v$. $\left[\mathbb{Z}_{2^{n}}^{n_{T}} / C_{0}\right]$ denotes a set of cosets representatives, one for each coset. In this paper, the coset representatives are $\left[\mathbb{Z}_{2^{n}}^{n_{T}} / C_{0}\right]=\mathbb{Z}_{2^{n-1}}^{n_{T}} \bmod 2$. So, the group $\mathbb{Z}_{2^{n}}^{n_{T}}$ is decomposed in $2^{n_{T}(n-1)}$ cosets.

Besides, it is possible to create $n$ sets of these cosets. For a $2^{n}$-PSK modulation and $n_{T}$ transmit antennas, the first set of cosets is $E_{0}=C_{0}=2^{n-1} \mathbb{Z}_{2}^{n_{T}}$. The other sets $E_{i}$ of cosets with $1 \leq i \leq n-1$ are defined by

$$
E_{i}=\bigcup_{g_{i}}\left(g_{i}+C_{0}\right) \text {, with } g_{i} \in 2^{n-i-1} \mathbb{Z}_{2^{i}}^{n_{T}} \backslash 2^{n-i} \mathbb{Z}_{2^{i-1}}^{n_{T}}
$$

Each coset $C_{p}=p+C_{0} \in E_{i}$ with $0 \leq i \leq n-2$ is called 'relative to $q=2 p \in E_{i-1}$ '. 


\subsection{Coset partitioning}

Calderbank et al. give an alternative to the set partitioning. It is based on the observation that the points of the constellation of a convolutional encoder can be regarded as a finite set of points from a group [11]. The group is partitioned into cosets. The Euclidean distances between the elements of the cosets are maximized. The coder selects at each time one coset. In each coset, it selects one element. It is possible to adapt the works of Ungerboeck and Calderbank et al. for the STTCs. In fact, the MIMO constellation can be seen as a finite subgroup of $\mathbb{Z}_{2^{n}}^{n_{T}}$. The goal of this method is that all the MIMO symbols originating from or merging to the same state belong to the same coset. The elements of these cosets must be separated by the largest Euclidean distances. Thus, for a $2^{n}$-PSK STTC with $2^{n \nu}$ states, the generator matrix $G$ is constituted by $\nu+1$ blocks of $n$ columns. To design the STTCs with the coset partitioning, each block $i$ must generate a subgroup. Thus, the following rules must be respected:

- For each block $k$, its first column $G_{1}^{k}$ must belong to $C_{0}$ with $1 \leq k \leq \nu+1$.

- The columns $G_{i}^{k}$ with $2 \leq i \leq n$ and $1 \leq k \leq \nu+1$ must belong to the cosets relative to an element generated by the first $(i-1)$ columns or in $C_{0}$.

After the choice of all columns of $\boldsymbol{G}$, it is possible to permute the columns and the lines of each block to obtain codes which respect the coset partitioning. Many subgroups exist and we show in the next section how to select the optimal elements to create the best generator matrices $\boldsymbol{G}$.

\subsection{Design example: 4 -PSK 4 states STTC with $n_{T}$ trans- mit antennas}

The MIMO symbols belong to $\mathbb{Z}_{4}^{n_{T}}$. This group can be divided in 2 subsets: $E_{0}=C_{0}$ and $E_{1}=\bigcup\left(g_{i}+C_{0}\right)$ with $g_{i} \in \mathbb{Z}_{2}^{n_{T}} \backslash[0 \ldots 0]^{T}$. The generator matrix $G$ has 2 blocks of 2 columns: $B_{1}=\left[G_{1}^{1}, G_{2}^{1}\right]$ and $B_{2}=\left[G_{1}^{2}, G_{2}^{2}\right]$ which give $\boldsymbol{G}=\left[G_{1}^{1} G_{2}^{1} \mid G_{1}^{2} G_{2}^{2}\right]$. If the coset partitioning is used to design a STTC, the columns of each block of $\boldsymbol{G}$ generates a subgroup of $\mathbb{Z}_{4}^{n_{T}}$. We note $\Lambda_{\nu+1}$ the set of MIMO symbols generated by the coder. It is possible to represent it with the trellis form. Fig. 3 shows the 4-PSK 4 states STTC where $\Lambda_{1}^{F}=\left\{\boldsymbol{y}_{0}, \boldsymbol{y}_{1}, \boldsymbol{y}_{2}, \boldsymbol{y}_{3}=\boldsymbol{y}_{1}+\boldsymbol{y}_{2} \bmod 4\right\}$ and $\Lambda_{1}^{M}=\left\{\boldsymbol{y}_{0}, \boldsymbol{y}_{4}, \boldsymbol{y}_{8}, \boldsymbol{y}_{12}=\boldsymbol{y}_{4}+\boldsymbol{y}_{8} \bmod 4\right\}$ are subgroups of $\mathbb{Z}_{4}^{n_{T}}$. Each coset of $\Lambda_{\nu+1} / \Lambda_{1}^{F}$ or of $\Lambda_{\nu+1} / \Lambda_{1}^{M}$ corresponds respectively to the set of MIMO symbols originating from the same state and the set of MIMO symbols merging into the same state. Thus, the generator matrix is $\boldsymbol{G}=\left[\boldsymbol{y}_{1} \boldsymbol{y}_{2} \mid \boldsymbol{y}_{4} \boldsymbol{y}_{8}\right]$. The rule 2 of Ungerboeck's method is: transitions originating from the same state or merging into the same state in the trellis should be assigned subsets which contain signal points separated by the largest Euclidean distances. In order to exploit the coset partitioning, the Euclidean distances between the elements of respectively $\Lambda_{1}^{F}$ and $\Lambda_{1}^{M}$ must be maximized thanks to the selection of $\left\{y_{1}, y_{2}\right\}$ and $\left\{y_{4}, y_{8}\right\}$.

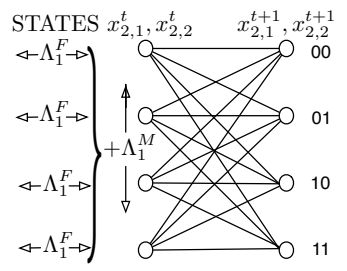

Fig. 3. 4-PSK 4 states STTC with cosets

Since each block of $n$ elements ( $n=2$ for 4-PSK) of $G$ generates a subgroup of $\mathbb{Z}_{2^{n}}^{n_{T}}$, the rule 1 of Ungerboeck's method is respected.

\subsection{Design example: 4-PSK 16 states STTC with $n_{T}$ transmit antennas}

The MIMO symbols belong to $\mathbb{Z}_{4}^{n_{T}}$. This group can be divided in 2 sets of cosets: $E_{0}=C_{0}, E_{1}=\bigcup\left(g_{i}+C_{0}\right)$ with $g_{i} \in \mathbb{Z}_{2}^{n_{T}} \backslash[0 \ldots 0]^{T}$. The matrix $G$ has 3 blocks of 2 columns: $B_{1}=\left[G_{1}^{1}, G_{2}^{1}\right], B_{2}=\left[G_{1}^{2}, G_{2}^{2}\right]$ and $B_{3}=\left[G_{1}^{3}, G_{2}^{3}\right]$ which give $\boldsymbol{G}=\left[G_{1}^{1} G_{2}^{1}\left|G_{1}^{2} G_{2}^{2}\right| G_{1}^{3} G_{2}^{3}\right]$. In the case of a 4-PSK 16 states STTC designed with coset partitioning, the trellis can be represented as shown in Fig. 4.

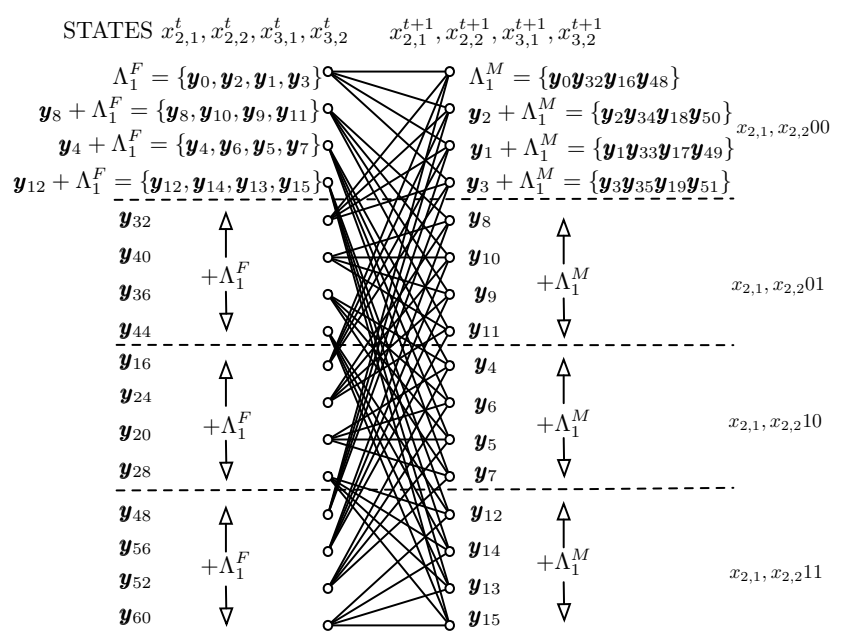

Fig. 4. 4-PSK 16 states STTC

On the left side of the trellis, there are the MIMO symbols $\boldsymbol{y}_{i}$ with $0 \leq i \leq 63$ originating from the same states are represented. On the right side of the trellis, we have the MIMO symbols merging into the same states. In this case, the generator matrix has 3 blocks of 2 columns $G=$ $\left[\boldsymbol{y}_{1} \boldsymbol{y}_{2}\left|\boldsymbol{y}_{4} \boldsymbol{y}_{8}\right| \boldsymbol{y}_{16} \boldsymbol{y}_{32}\right]$ as shown in Fig. 4. There are two steps to design $G$ : 
- The first step is to select the elements of the first and the last block. The first block and the last block of each generator matrix generate respectively the subgroups $\Lambda_{1}^{F}=\left\{\boldsymbol{y}_{0}, \boldsymbol{y}_{1}, \boldsymbol{y}_{2}, \boldsymbol{y}_{3}=\boldsymbol{y}_{1}+\boldsymbol{y}_{2} \bmod 4\right\}$ and $\Lambda_{1}^{M}=\left\{\boldsymbol{y}_{0}, \boldsymbol{y}_{16}, \boldsymbol{y}_{32}, \boldsymbol{y}_{48}=\boldsymbol{y}_{16}+\boldsymbol{y}_{32} \bmod 4\right\}$. Each coset of $\Lambda_{\nu+1} / \Lambda_{1}^{F}$ and of $\Lambda_{\nu+1} / \Lambda_{1}^{M}$ corresponds to the transitions originating from the same state and the transitions merging into the same state. The Euclidean distances between the elements of each subgroup created by the columns of these blocks must be maximized.

- The second step is the selection of $B_{2}$. The vectors of $B_{2}$ must also generate a subgroup. So, it is possible to represent a simplified trellis as shown in Fig. 5 where the new points $x_{3,1} x_{3,2}=\{00,01,10,11\}$ of Fig. 5 are defined as a set of 'states of level 2'. Each state of level 2 is constituted by 4 real states $\left\{00 x_{3,1} x_{3,2}, 01 x_{3,1} x_{3,2}, 10 x_{3,1} x_{3,2}, 11 x_{3,1} x_{3,2}\right\}$. The new lines correspond to the 'transitions of level 2 ' which are constituted by 4 real transitions. If a coset partitioning is used, we have $\Lambda_{2}^{F}=\Lambda_{1}^{F} \cup\left(\Lambda_{1}^{F}+\boldsymbol{y}_{4}\right) \cup$ $\left(\Lambda_{1}^{F}+\boldsymbol{y}_{8}\right) \cup\left(\Lambda_{1}^{F}+\boldsymbol{y}_{12}\right)$ which is a subgroup of $\mathbb{Z}_{2^{n}}^{n_{T}}$ (with $\boldsymbol{y}_{4}+\boldsymbol{y}_{8}=\boldsymbol{y}_{12} \bmod 4$ ). Thus, a partition chain is obtained: $\Lambda_{\nu+1} / \Lambda_{2}^{F} / \Lambda_{1}^{F}$. The choice of $y_{4}$ and $y_{8}$ must maximimize the minimum Euclidean distance between two different elements of $\Lambda_{2}^{F}$.

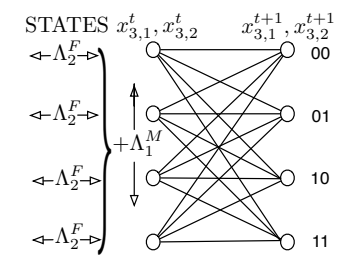

Fig. 5. Simplification of trellis 4-PSK 16 states STTC with cosets

It is possible to make a chain partition with the MIMO symbols merging into the different levels of states: $\Lambda_{\nu+1} / \Lambda_{2}^{M}$ $/ \Lambda_{1}^{M}$. Each element of $\Lambda_{\nu+1} / \Lambda_{2}^{M}$ corresponds to one coset of MIMO symbols merging into a same state of level 2. As shown in Fig. $5, \Lambda_{2}^{M}$ is given by: $\Lambda_{2}^{M}=\Lambda_{1}^{M}+\Lambda_{1}^{F}$. Thus, $\Lambda_{2}^{M}$ is already defined by the previous selections.

At the end, we permute the elements of each block to find the codes with the best trace.

\subsection{Design example: 8-PSK 8 states STTC with 4 trans- mit antennas}

The MIMO symbols generated by the 8-PSK 8 states STTCs with 4 transmit antennas belong to $\mathbb{Z}_{8}^{4}=E_{0} \cup E_{1} \cup E_{2}$.

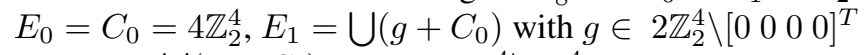
and $E_{2}=\bigcup\left(h+C_{0}\right)$ with $h \in \mathbb{Z}_{4}^{4} \backslash 2 \mathbb{Z}_{2}^{4}$. The generator matrix $G$ is constituted by 2 blocks of 3 columns. To generate
$G$ we can proceed as follows. We must select 3 elements of $\mathbb{Z}_{8}^{4}$ for the 2 blocks which create subgroups of $\mathbb{Z}_{8}^{4}$. The minimum Euclidean distance between two different elements of each group must be maximized. In fact, these two subgroups correspond to the transitions originating from the same state and to the transitions merging into the same state. An 'optimal' subgroup of $\mathbb{Z}_{8}^{4}$ is generated thanks to one element of $C_{0}=E_{0}$, one element of $E_{1}$ and one element of $E_{2}$. After the selection of two good blocks, we search the code with the optimal trace. We obtain: $\boldsymbol{G}=\left[\begin{array}{ccc|ccc}4 & 2 & 1 & 0 & 0 & 4 \\ 4 & 6 & 3 & 0 & 4 \\ 0 & 4 & 2 & 4 & 4 \\ 0 & 0 & 4 & 2 & 3 \\ \hline & & 0 & 6 & 6 & 7\end{array}\right]$.

Remark 1: Some codes may have first $i$ null columns in the $(\nu+1)^{t h}$ block with $0<i<n$. The number of states is $2^{n \nu-i}$. In this case, the columns which generate the subgroup $\Lambda_{1}^{M}$ (MIMO symbols which merge into a same state) are the first $i$ columns of $\nu^{\text {th }}$ blocks and the last $n-i$ columns of the $(\nu+1)^{t h}$ block.

\section{NEW CODES AND PERFORMANCE ANALYSIS}

In Table 1, Chen's 4-PSK codes and new corresponding codes are shown for 3 transmit antennas/32 states and 4 transmit antennas/64 states. The new code with 4 transmit antennas has a higher value of the minimum trace than that of the Chen's equivalent code. The new code with 3 transmit antennas has a trace equal to the trace of the Chen's corresponding code, but, the minimum Euclidean distance between two differents elements generated by each block of the new code are higher. In Table 2, Chen's 8-PSK 8 states codes and new corresponding codes are shown for 3 and 4 transmit antennas. The new code with 3 transmit antennas has a trace equal to the trace of the Chen's corresponding code and the code with 4 transmit antennas has a trace lower than the Chen's corresponding code. We compute the minimum Euclidean distance between the elements generated by each block. Their minimum value is higher than that of the Chen's corresponding code.

\begin{tabular}{|c|c|c|c|c|c|c|c|c|c|c|c|}
\hline$n_{T}$ & $2^{\nu n}$ & Code & \multicolumn{8}{|c|}{$G$} & Trace \\
\hline \multirow[t]{2}{*}{3} & \multirow[t]{2}{*}{32} & Chen & $\begin{array}{l}0 \\
2 \\
2\end{array}$ & $\begin{array}{l}2 \\
2 \\
0\end{array}$ & $\begin{array}{l}2 \\
3 \\
3\end{array}$ & $\begin{array}{l}1 \\
2 \\
2\end{array}$ & $\begin{array}{l}1 \\
2 \\
2\end{array}$ & $\begin{array}{l}2 \\
3 \\
1\end{array}$ & $\begin{array}{l}0 \\
0 \\
0\end{array}$ & $\begin{array}{l}2 \\
0 \\
0\end{array}$ & 24 \\
\hline & & New 1 & $\begin{array}{l}0 \\
2 \\
2\end{array}$ & $\begin{array}{l}2 \\
1 \\
1\end{array}$ & $\begin{array}{l}2 \\
2 \\
2\end{array}$ & $\begin{array}{l}1 \\
1 \\
3\end{array}$ & $\begin{array}{l}2 \\
0 \\
2\end{array}$ & $\begin{array}{l}1 \\
2 \\
1\end{array}$ & $\begin{array}{l}0 \\
0 \\
0\end{array}$ & $\begin{array}{l}1 \\
2 \\
3\end{array}$ & 24 \\
\hline \multirow[t]{2}{*}{4} & \multirow[t]{2}{*}{64} & Chen & $\begin{array}{l}0 \\
2 \\
2 \\
1\end{array}$ & $\begin{array}{l}2 \\
2 \\
0 \\
2\end{array}$ & $\begin{array}{l}3 \\
1 \\
0 \\
2\end{array}$ & $\begin{array}{l}2 \\
2 \\
2 \\
0\end{array}$ & $\begin{array}{l}3 \\
3 \\
2 \\
2\end{array}$ & $\begin{array}{l}0 \\
0 \\
3 \\
1\end{array}$ & $\begin{array}{l}3 \\
2 \\
1 \\
3\end{array}$ & $\begin{array}{l}2 \\
0 \\
1 \\
2\end{array}$ & 38 \\
\hline & & New 2 & $\begin{array}{l}2 \\
0 \\
2 \\
2\end{array}$ & $\begin{array}{l}3 \\
2 \\
3 \\
1\end{array}$ & $\begin{array}{l}0 \\
2 \\
0 \\
2\end{array}$ & $\begin{array}{l}2 \\
1 \\
2 \\
1\end{array}$ & $\begin{array}{l}2 \\
2 \\
0 \\
2\end{array}$ & $\begin{array}{l}3 \\
1 \\
2 \\
3\end{array}$ & $\begin{array}{l}0 \\
2 \\
2 \\
2\end{array}$ & $\begin{array}{l}2 \\
3 \\
3 \\
1\end{array}$ & 40 \\
\hline
\end{tabular}

Table 1. New 4-PSK code based on the Euclidean distance criteria

The performance of each code is evaluated by simulation in a slow Rayleigh fading channel. The channel fading coefficients are independent samples of a complex Gaussian process with zero mean and variance 0.5 per dimension. These channel coefficients are assumed to be known at the decoder. Each frame consists of 130 4-PSK or 8-PSK symbols. For the simulation, there are 2 receive antennas. The decoding is 


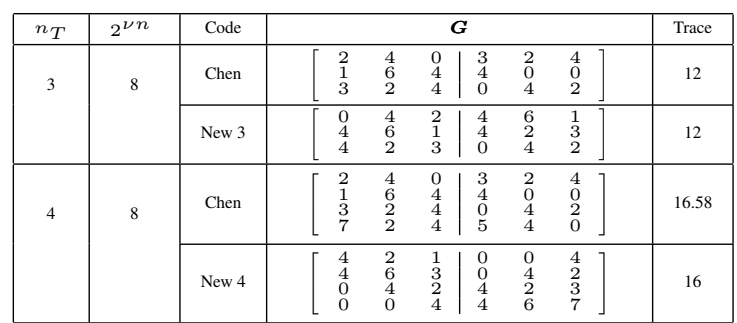

Table 2. New 8-PSK codes based on the Euclidean distance criterion

performed by the Viterbi's algorithm. Fig. 6 shows the performance of the 4-PSK 64 states codes for 3 and 4 transmit antennas given in Table 1. Similary, Fig. 7 shows the performance of the new 8-PSK codes and the corresponding Chen's codes of Table 2.

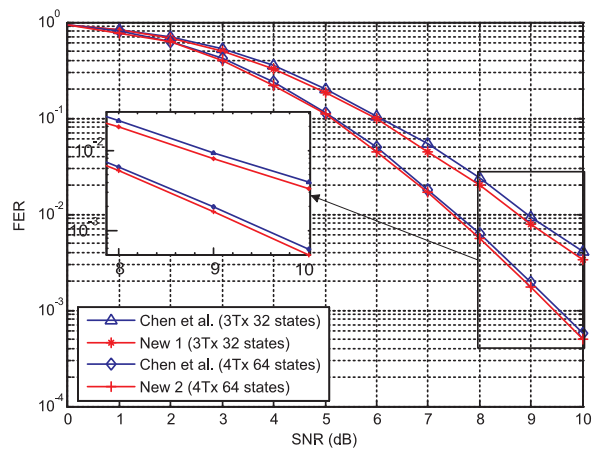

Fig. 6. Performance of 4-PSK 64/32 states STTCs

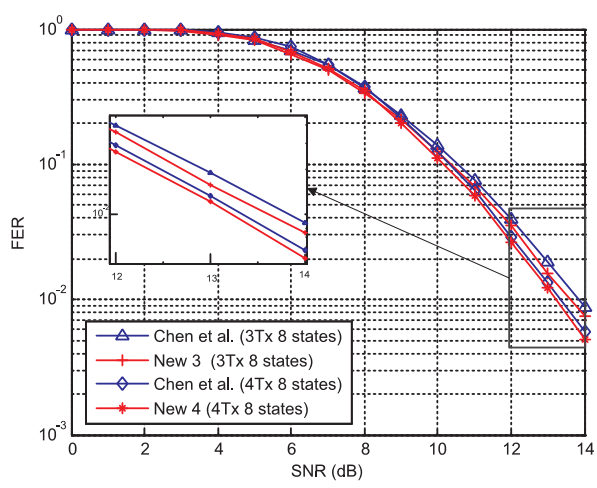

Fig. 7. Performance of 8-PSK 8 states STTCs

\section{CONCLUSION}

A new and fast method to design easily the best $2^{n}$-PSK STTCs with $n_{T}$ transmit antennas has been presented in this paper. This method reduces significantly the number of possible codes which must be analysed with an exhaustive search.
The proposed method is based on the set partitioning proposed by Ungerboeck and an extension of the work proposed by Calderbank. This method is called 'coset partitioning'. Thanks to this method, new 4-PSK and 8-PSK codes are obtained. It is shown that these new codes outperform the best known codes.

\section{REFERENCES}

[1] V. Tarokh, N. Seshadri, and A.R. Calderbank, "Spacetime codes for high data rate wireless communication: Performance criterion and code construction," IEEE Trans. Inform. Theory, vol. 44, no. 2, pp. 744-765, Mar. 1998.

[2] Z. Chen, J. Yuan, and B. Vucetic, "Improved space-time trellis coded modulation scheme on slow fading channels," Electron. Lett., vol. 37, no. 7, pp. 440-441, Mar. 2001.

[3] Z. Chen, B. Vucetic, J. Yuan, and Ka Leong Lo, "Spacetime trellis codes with two, three and four transmit antennas in quasi-static flat fading channels," in Proc. IEEE ICC, May 2002, vol. 3, pp. 1589-1595.

[4] S. Baro, G. Bauch, and A. Hansmann, "Improved codes for space-time trellis-coded modulation," IEEE Commun. Lett., vol. 4, no. 1, pp. 20-22, Jan. 2000.

[5] B. Abdool-Rassool, M.R. Nakhai, F. Heliot, L. Revelly, and H. Aghvami, "Search for space - time trellis codes: novel codes for rayleigh fading channels," IEE Proc.Commun., vol. 151, no. 1, pp. 25-31, Feb. 2004.

[6] G. Ungerboeck, "Channel coding with multilevel/phase signals," IEEE Trans. Inform. Theory, vol. 28, pp. 5567, Jan. 1982.

[7] G. Ungerboeck, "Trellis-coded modulation with redundant signal sets part I: Introduction," Comm. Magazine, IEEE, vol. 25, pp. 5-11, Feb. 1987.

[8] G. Ungerboeck, "Trellis-coded modulation with redundant signal sets part II: State of the art," Comm. Magazine, IEEE, vol. 25, pp. 12-21, Feb. 1987.

[9] C. Liao and V.K. Prabhu, "Improved code design criteria for space-time codes over quasi-static flat fading channels," in Proc. IEEE SPAWC, June 2005, pp. 7-11.

[10] Jeffrey O. Colemann, "Coset decomposition in lattices yields sample-block number systems," Proc. IEEE ISCAS, vol. II, pp. 688-691, May 2002.

[11] A. Calderbank and N. Sloane, "New trellis codes based on lattices and cosets," IEEE Trans. Inform. Theory, vol. 33, pp. 177-195, Mar. 1987. 